\title{
Medical decision making for cardiac MRI "5D Model of the Descending Aorta for the Detection of Severe Stenosis"
}

\author{
Houneida Sakly ${ }^{1}$, Mourad Said ${ }^{2}$, and Moncef Tagina ${ }^{1}$ \\ ${ }^{1}$ ENSI \\ ${ }^{2}$ International Center Carthage Medical
}

September 11, 2020

\begin{abstract}
Purpose: Depict an accurate 5D ( $\mathrm{x}, \mathrm{y}, \mathrm{z}$, Time flow dimension) model for medical decision medical. The time-dependent instantaneous pressure gradient across the aorta proposed advanced measures for the detection of severe stenosis (Flow rate, Reynolds Number, velocity ... ) Methods: A 74 cardiac MRI scan was performed and 3057 images for a patient aged 10 years, with a record of congenital valve and valvular aortic stenosis in tight MRI and coarctation (operated and then dilated) in the context of shone syndrome. Results: The occlusion rate was estimated of $80.5 \%$. The stenosis zone is assessed about $15 \mathrm{~mm}$ and extended over $10 \mathrm{~mm}$. The fluid solver (NS) extract a negative pressure value of $-3,735$ e005 [Pa], a remarkable fall of the flux mass was detected with $-0,0050(\mathrm{~kg} / \mathrm{s})$ during the first 10 iterations, as well as high blood turbulence in vortex field lines and low geometry Reynolds cells. The fifth dimension was treated separately to analyze velocity at the aortic valve with shone syndrome for negative velocity identification $(-81.4 \mathrm{~cm} / \mathrm{s})$. Conclusion: The results of $5 \mathrm{D}$ aortic modeling are considered promising with its representation of its physical and anatomical properties.
\end{abstract}

5D Modeling of the Descending Aorta for the Detection of Severe Stenosis

Houneida Sakly ${ }^{1}$, Mourad Said ${ }^{2}$, Moncef Tagina ${ }^{1}$

${ }^{1}$ COSMOS Laboratory -National Institute of Computer Sciences - Campus University of Mannouba -Tunisia

${ }^{2}$ Radiology and Medical Imaging Unit, International Center Carthage Medical, Tourist Area "JINEN EL OUEST"-5000 Monastir, Tunisia

houneida.sakly@esiee.fr, mouradsaid@yahoo.fr, moncef.tagina@ensi-uma.tn

*Corresponding author: Houneida Sakly, COSMOS Laboratory -National Institute of Computer Sciences, University of Mannouba, Tunisia

Abstract:

Purpose:

Depict an accurate 5D (x, y,z,Time+flow dimension) model for medical decision medical. The time-dependent instantaneous pressure gradient across the aorta proposed advanced measures for the detection of severe stenosis (Flow rate, Reynolds Number, velocity ... )

Methods:

A 74 cardiac MRI scan was performed and 3057 images for a patient aged 10 years, with a record of congenital valve and valvular aortic stenosis in tight MRI and coarctation (operated and then dilated) in the context of shone syndrome. 


\section{Results:}

The occlusion rate was estimated of $80.5 \%$. The stenosis zone is assessed about $15 \mathrm{~mm}$ and extended over $10 \mathrm{~mm}$. The fluid solver (NS) extract a negative pressure value of $-\mathbf{3 , 7 3 5}$ e005 $[\mathrm{Pa}]$, a remarkable fall of the flux mass was detected with $-0,0050(\mathrm{~kg} / \mathrm{s})$ during the first 10 iterations, as well as high blood turbulence in vortex field lines and low geometry Reynolds cells. The fifth dimension was treated separately to analyze velocity at the aortic valve with shone syndrome for negative velocity identification $(-81.4 \mathrm{~cm} / \mathrm{s})$.

Conclusion: The results of 5D aortic modeling are considered promising with its representation of its physical and anatomical properties.

Key words: Fluid Solver, 5D model of the aorta, pressure, Flow rate, Reynolds Number, velocity, MRI

\section{Introduction}

In this paper the concept and the modeling of the 5D cardiac system was described (3D model + temporal dimension functional dimension of the flow) detailed in our research work mentioned in (Sakly et al., 2019) and (Sakly et al., 2020). This strategy consists in the reconstructing of a 3D geometry of the descending aorta and diffuse a 2D viscous laminar fluid to detect the zones of narrowing. Blood flow is impeded at the site of the stenosis in the narrowed aorta, which hampers the transport of blood cells and subsequently causes turbulence of the wall of the internal aorta. However, the behavior of blood flow depends on various other parameters, including thermo-physical properties such as viscosity, surface tension, and wettability (Sikarwar et al., 2016). Different mathematical models of blood rheology have been studied over the last decades and on the basis of these studies, various hidden characteristics are discovered such as the behavior of the fluid and the nature of the flow.(Roy et al., 2017). Therefore, defining the value of wall pressure and tension stress of the wall in the arteries is a vital issue for medical experts. Many researchers have reported pulsed flow behavior and its fluctuations are continuously damped, which can be attributed to the elasticity of the blood arteries. Blood-artery disease plays an important role in hemodynamics because it disrupts the flow pattern that causes a change in wall pressure and shear stress in the arteries (Cebral et al., 2011). Previously, a large part of published studies (Thomas \& Sumam, 2016) focused on the physiological causes of blood-related disease. However, a very small number is an incredible step to understand the underlying physics of the disease in order to understand the cause and thus pave the way for less invasive and more sustainable techniques for their processing. Current imaging techniques do not allow doctors to know the parietal pressure and the parietal stress exerted by the blood on the inner periphery of the artery. The revolutionary development of physiological modeling of pathology and cardiovascular physiology paves the way for bridging the gap. Recent developments in the field of Computational Fluid Dynamics (CFD) have the ability to simulate blood flow in a geometry of the heart structure. It is a less invasive method; a computer can show you the flow pattern of blood for a different disease artery. As a result, CFD has now become a clinical diagnostic tool for the medical practitioner in the field of congenital heart valve, coronary, myocardial and peripheral vascular diseases.(Xiong et al., 2011). However, the CFD module relies on the precise definition of geometric and flow boundary conditions and requires non-clinical expertise, engineering tools, powerful computer systems and a large number of calculations. Because of these requirements and limitations, it is difficult to include CFDs in routine clinical practices. Alternatively, the viscous dissipation can be calculated using the viscous term of the Navier-Stokes equation (Cibis et al., 2015). This approach bypasses the need for pressure and requires only the blood flow velocities inside, which can be obtained noninvasively in $4 \mathrm{D}$ flow $\mathrm{MRI}$.

Our main contribution is to present a numerical simulation of a laminar blood flow in 3D aortic modeling in the presence of a left subclavian aortic coarctation as well as an analytical study is explained to study the impact of a solver of dynamic fluid on the detection of aortic stenosis. 


\section{II.Methods and Materiels}

A 74 cardiac MRI scan was performed and 3057 images for a patient aged 10 years, with a record of congenital valve and valvular aortic stenosis in tight MRI and coarctation (operated and then dilated) in the context of shone syndrome.

Technically, reconstruction and segmentation of the descending aorta was performed with

- 44 TRICKS angiographic slices in dynamic acquisition on the thoracic aorta

- Injection perfusion sequences after injection

- Sequences ciné-fiesta T2 short-axis 4 cavities

- Subsequent infusion sequences Short-axis

Clinical diagnostic report of $L V$ :

Ascending thoracic aorta without parietal abnormality. Its main measurements are:

- Tricuspid valve. Aortic ring with $8.5 \mathrm{~mm}$ diameter

- Aortic stenosis at $0.42 \mathrm{~cm} 2$ with reduction of sigmoid opening at $5 \mathrm{~mm}$

- At the sino-tubular junction: $25 \mathrm{~mm}$

- $1 / 3$ medium of the ascending aorta: $18 \mathrm{~mm}$

- Horizontal aorta: $16 \mathrm{~mm}$

- Size disparity with aortic stenosis at the isthmic level extended over $10 \mathrm{~mm}$ long, reducing about $65 \%$ of its lumen by $6 \mathrm{~mm}$ in diameter.

- Mitral valve of normal diameter $2.5-4.3 \mathrm{~cm} 2$

- The mass of VG tele-diastolic $90 \mathrm{~g}$ and tele-diastolic $70 \mathrm{~g}$

- The systolic ejection function, estimated according to the $82 \%$ contour method

Features of TRICKS cuts and acquisition protocols

- Magnetic field: 1.5 Tesla

- Acquisition time: 1.2 seconds / Repetition time: 3.3 seconds

- Diameter of reconstruction of the cuts: $370 \mathrm{~cm} 2$

- Angle of acquisition $=30$ degrees

- Acquisition matrix: 0/300/224/0

- Sections orientation matrix: $-0.0393457 \backslash 0.99917 \backslash-0.0105243 \backslash 0.283665 \backslash 0.00106985 \backslash-0.958923$

- Cutting position matrix: $-1.15748 \backslash-152.042 \backslash 358.794$

- Number of time positions: 12

- Scanning Rentals: -43.95322037

- Space between pixels: $0.7227 \backslash 0.7227$ with allocation of 16 bits of memory

\section{III.Results}

\section{III.1. Model of the geometry of the descending aorta in 3D}

In this section, the first phase of our 5D model which consists of defining the geometry of the descending aorta in 3D was proceeded. This step states on the reconstruction of the aortic model from the TRICKS sections. The Multiplanar reconstruction in Figure.6 is a crucial step to carry out 3D reconstruction for the descending aorta presented in axial plane in Fig.1 and Fig.2:

A threshold to the areas of interest that defines the active mask was applied based a low limit and a high limit. The mask contains pixels with a value between the two threshold limits. The upper and lower thresholds are limited to the maximum and minimum intensity. The updates according to the defined limits gives a 
reconstruction of the model of the aorta in 3D based on the Multiplanar reconstruction of the TRICKS cuts in Figure.3:

The refinements of the three-dimensional reconstructions obtained depends essentially on the conditions and quality of acquisition of cuts, thickness of the cuts, the distance between two successive acquisition slices and the quality of the segmentation and the reconstruction algorithm used. The final geometry is shown in Figure.4:

Mathematical modeling and simulation environment presentation is detailed in (Wilhelm et al., 2016) with precision analysis for IGS models in (Ray et al., 2017). The second phase consists of decomposing this IGS model into 3 elements: body, inlet, outlet as shown in Figure.5:

The geometry of the aorta has the following characteristics: length along the x-axis $=3.6339 \mathrm{e}-002$ length along the $\mathrm{y}$-axis $=5.66596 \mathrm{e}-002 \mathrm{~m}$, length along the $\mathrm{z}$-axis $=8.4559 \mathrm{e}-002 \mathrm{~m}$ with a total volume $=2.5915 \mathrm{e}-$ $005 \mathrm{~m}^{3}$, number of nodes $=593223$, and number of elements $=3168417^{2}$. The number of faces of the body (wall) is equal to 449 with total area of $5.6453 \mathrm{e}-003 \mathrm{~m}^{2}$. The result of the mesh is presented in Figure.6

\section{III.2. Setting of the solution}

To solve the 2D Navier-Stokes equation the configuration of this fluid solver was based on the pressure with absolute velocity formation in stable time. Our fluid will be blood with density of $1056(\mathrm{~kg} / \mathrm{m} 3)$ and viscosity of $6(\mathrm{~kg} / \mathrm{m} 3)$. Boundary conditions must be zero in the aortic model. The execution of the laminar flow model defines velocities and pressure in the viscous flow field (the Navier-Stokes equation) to solve the displacement of the internal facet of the $3 \mathrm{D}$ model of the aorta.

In the momentum that governs fluid equations, velocity and pressure the parameters are coupled. There are two main types of methods for solving the discrete time equation algebraic equations: the coupled method and the separate method. A coupled method is characterized by the simultaneous solution of velocity and pressure parameters. Due to the low computing efficiency and the large memory requirement, it has not been widely applied in engineering problems. Coupled methods have been widely used for calculating compressible flows, while separate methods have been preferred for calculating incompressible flows. Unlike a coupled solution, a separate method solves the velocity and pressure fields separately or consecutively. It presents the advantages of reducing the computer's memory and computing time, making it more efficient for analyzing incompressible environment simulation fluids as it is indicated in our case for aortic stenosis modeling. (Wang et al., 2018). The pressure analysis for our model is presented in Figure.7.

It could be concluded from this illustration that the pressure of the entrance and exit is negligible compared to the body outside and inside the model of the stenotic aorta. The pressure intersection $(\mathrm{Pa})$ between these 2 zones (inside and outside) of the wall at a critical point in the position of $15 \mathrm{~mm}$ shows a remarkable overlap between the red and blue part which explains why we can have a turbulence because of the strength of the parietal wall exerted by the external and internal body. The location of blood flow in a case of aortic dissection with a complicated geometric feature, qualitatively and quantitatively, based on the evolution of vortex structures and their interaction in the narrowing region throughout a cardiac cycle can give an index on the presence of a stenosis from $15 \mathrm{~mm}$ length of the aortic segment.

The position of $15 \mathrm{~mm}$ there is a very high vortex magnitude which reaches $(8000001 / \mathrm{s})$ as well as a mass of negative fluxes during the first 6 iterations during solver calculation. This reflux shows that it occludes in a segment of the aorta. These indices support the results reported in the clinical assessment with isthmic extensor aortic stenosis extended over $10 \mathrm{~mm}$ in length, reducing approximately $65 \%$ of its lumen by $6 \mathrm{~mm}$ in diameter, compared to what we estimated there is $15 \mathrm{~mm}$ wide aortic stenosis, a $5 \mathrm{~mm}$ error rate is detected.

\section{III.3. Solution with ANSYS-Fluent}

CFD predicts the overall size of the stagnation and shunt zones, but underestimates the length of the current line and the changes in speed due to the flow in the aorta, a whole range of physics has been included beyond 
simple structural simulations: fluid flow, electromagnetic, thermal(Zaripov et al., 2018),(Tora \& Dahlquist, 2015),(Hosseini \& Vahedi Tafreshi, 2012). However, the quality of the measurement needs to be improved for quantitative validation of CFD results and the search for flow effects such as tortuosity and laminar flow behavior. At the same time, the results of the CFD simulations are interpreted not only in terms of pressure drop but in all aspects of flow such as vector orientation and velocity, current lines, areas of stagnation of blood flow.(Schlanstein et al., 2015) . This concept of time-based flow is considered in 3D modeling of the stenosing aorta to obtain the 5D format as shown in Figure.8.

Due to the fiber of this model, the fluid flow is deflected, resulting in a sinuous orientation of the flow lines which shows a three-dimensional flow around the wall of the aorta, mainly directed from the inlet to the outlet. The area of stenosis in the middle indicated by lower speeds can be seen in Figure.9.

Focusing on the velocity in the stenosis part, we notice that the flow field lines are delayed and decreased from 6.034 to $1.207 \mathrm{e} 001$ to $1.810 \mathrm{e} 001$ [ms-1]. What is interesting is the detection of a negative pressure in the value narrowing zone is equal to -3.735 e005 $[\mathrm{Pa}]$ in Figure.10.

\section{Discussion}

In this paper we presented a medical decision support approach for cardiac imaging in MRI that states on the 5D modeling (3D anatomical structure, temporal dimension + blood flow dimension) for the analysis of a promising case of aortic coarctation with severe valve narrowing. Our object of interest was a segment of the descending aorta for prediction, identifying the presence of aortic stenosis. The results show, in the first stage, that the 3D modeling gives a very interesting index for the experts which makes it possible to estimate the occlusion rate of $80.5 \%$ compared to what was manifested in the clinical assessment with a rate of $82 . \%$. The measurements extracted for the thickness and the local curvature with respect to the geometry of the aorta mark the zone of stenosis and the degree of deflection of the narrowing portion. In the second stage, the presentation of the 5D approach is done through the combination of the 3D model and the size of the circulating blood flow as a function of time. The experiments that were done for the fifth dimension provides high accuracy for the location of the stenosis zone with $15 \mathrm{~mm}$ in length compared to the clinical prognosis that indicates that aortic stenosis is extended over $10 \mathrm{~mm}$ These results are deduced from the solver of fluid (NS) which is based on the interpretation and observation of a negative pressure value of $-3,735 \mathrm{e} 005[\mathrm{~Pa}]$, a remarkable fall of the flux mass was detected with $-0,0050(\mathrm{~kg} / \mathrm{s})$ during the first 10 iterations, as well as high blood turbulence in vortex field lines and low geometry Reynolds cells. The fifth dimension was treated separately to analyze velocity at the aortic valve with shone syndrome for negative velocity identification $(-81.4 \mathrm{~cm} / \mathrm{s})$.

Conflict of Interest/Financial Disclosure: All authors confirm that no conflict of interest and no financial disclosure to declare

Acknowledgments: Many thanks go to the Radiology and Medical Imaging Unit, International Center Carthage Medical, that supported this work and to the medical staff for providing us with an access to the patients' archive and the administrative framework for the warm welcome in their team.

Funding sources : No finding resources received for this research work

\section{References}

Cebral, J. R., Mut, F., Raschi, M., Scrivano, E., Ceratto, R., Lylyk, P., \& Putman, C. M. (2011). Aneurysm rupture following treatment with flow-diverting stents: Computational hemodynamics analysis of treatment.AJNR. American Journal of Neuroradiology , 32 (1), 27-33. https://doi.org/10.3174/ajnr.A2398

Cibis, M., Jarvis, K., Markl, M., Rose, M., Rigsby, C., Barker, A. J., \& Wentzel, J. J. (2015). The effect of resolution on viscous dissipation measured with 4D flow MRI in patients with Fontan circulation: Evaluation using computational fluid dynamics.Journal of Biomechanics , 48 (12), 2984-2989. 
https://doi.org/10.1016/j.jbiomech.2015.07.039

Hosseini, S. A., \& Vahedi Tafreshi, H. (2012). Modeling particle-loaded single fiber efficiency and fiber drag using ANSYS-Fluent CFD code.Computers $\&$ Fluids , 66 , 157-166. https://doi.org/10.1016/j.complluid.2012.06.017

Ray, J., Rebischung, P., \& Griffiths, J. (2017). IGS polar motion measurement accuracy. Geodesy and Geodynamics , 8 (6), 413-420. https://doi.org/10.1016/j.geog.2017.01.008

Roy, M., Singh Sikarwar, B., Bhandwal, M., \& Ranjan, P. (2017). Modelling of Blood Flow in Stenosed Arteries. Procedia Computer Science , 115 , 821-830. https://doi.org/10.1016/j.procs.2017.09.164

Sakly, H., Mahmoudi, R., Akil, M., Said, M., \& Tagina, M. (2019). MOVING TOWARDS A 5D CARDIAC MODEL. Journal of Flow Visualization and Image Processing, 26 (1). https://doi.org/10.1615/JFlowVisImageProc.2018027194

Sakly, H., Said, M., Radhouane, S., \& Tagina, M. (2020). Medical decision making for 5D cardiac model: Template matching technique and simulation of the fifth dimension. Computer Methods and Programs in Biomedicine , 191 , 105382. https://doi.org/10.1016/j.cmpb.2020.105382

Schlanstein, P. C., Hesselmann, F., Jansen, S. V., Gemsa, J., Kaufmann, T. A., Klaas, M., Roggenkamp, D., Schröder, W., Schmitz-Rode, T., Steinseifer, U., \& Arens, J. (2015). Particle Image Velocimetry Used to Qualitatively Validate Computational Fluid Dynamic Simulations in an Oxygenator: A Proof of Concept. Cardiovascular Engineering and Technology , 6 (3), 340-351. https://doi.org/10.1007/s13239-015-0213-2

Sikarwar, B. S., Roy, M., Ranjan, P., \& Goyal, A. (2016). Automatic disease screening method using image processing for dried blood microfluidic drop stain pattern recognition. Journal of Medical Engineering $\mathcal{E}^{3}$ Technology , 40 (5), 245-254. https://doi.org/10.3109/03091902.2016.1162215

Thomas, B., \& Sumam, K. S. (2016). Blood Flow in Human Arterial System-A Review. Procedia Technology , 24, 339-346. https://doi.org/10.1016/j.protcy.2016.05.045

Tora, E., \& Dahlquist, E. (2015). CFD Ansys-Fluent Simulation of Prevention of Dioxins Formation Via Controlling Homogeneous Mass and Heat Transfer within Circulated Fluidized Bed Combustor. Energy Procedia , 75 , 130-136. https://doi.org/10.1016/j.egypro.2015.07.236

Wang, H., Wang, H., Gao, F., Zhou, P., \& Zhai, Z. (John). (2018). Literature review on pressure-velocity decoupling algorithms applied to built-environment CFD simulation. Building and Environment ,143 , 671-678. https://doi.org/10.1016/j.buildenv.2018.07.046

Wilhelm, M., Dedè, L., Sangalli, L. M., \& Wilhelm, P. (2016). IGS: An IsoGeometric approach for smoothing on surfaces. Computer Methods in Applied Mechanics and Engineering , 302 , 70-89. https://doi.org/10.1016/j.cma.2015.12.028

Xiong, G., Figueroa, C. A., Xiao, N., \& Taylor, C. A. (2011). Simulation of blood flow in deformable vessels using subject-specific geometry and spatially varying wall properties. International Journal for Numerical Methods in Biomedical Engineering , 27 (7), 1000-1016. https://doi.org/10.1002/cnm.1404

Zaripov, T. S., Rybdylova, O., \& Sazhin, S. S. (2018). A model for heating and evaporation of a droplet cloud and its implementation into ANSYS Fluent. International Communications in Heat and Mass Transfer , 97 , 85-91. https://doi.org/10.1016/j.icheatmasstransfer.2018.06.007

Figures Legends

Figure 1. Multiplanar reconstruction of TRICKS cuts for the aorta

Figure 2. Model of the descending aorta in axial plane

Figure 3. Reconstruction of the 3D model of the heart with the aorta 
Figure 4. Final reconstruction of the descending aorta model in $3 D$

Figure 5. Decomposition model of the aorta (a) in inlet (b), outlet (c) and wall (d)

Figure 6. Mesh of $3 D$ aorta

Figure 7. Calculation of static pressure for input of aortic model in 3D (inlet), outlet (outlet), model of aorta outside (wall), model of aorta inside (interior-face5)

Figure 8. Model $5 D$ of the aorta

Figure 9. Flow line visualization in the 5D aorta model (without the 3D inner wall)

Figure 10. Pressure de of the 5D model of the aorta (without the inner wall in 3D)

\section{Hosted file}

Figue1.docx available at https://authorea.com/users/351106/articles/475773-medical-decisionmaking-for-cardiac-mri-5d-model-of-the-descending-aorta-for-the-detection-of-severestenosis

\section{Hosted file}

Figure2 . docx available at https://authorea.com/users/351106/articles/475773-medical-decisionmaking-for-cardiac-mri-5d-model-of-the-descending-aorta-for-the-detection-of-severestenosis

\section{Hosted file}

Figure3. docx available at https://authorea.com/users/351106/articles/475773-medical-decisionmaking-for-cardiac-mri-5d-model-of-the-descending-aorta-for-the-detection-of-severestenosis

\section{Hosted file}

Figure4.docx available at https://authorea.com/users/351106/articles/475773-medical-decisionmaking-for-cardiac-mri-5d-model-of-the-descending-aorta-for-the-detection-of-severestenosis

\section{Hosted file}

Figure5. docx available at https://authorea.com/users/351106/articles/475773-medical-decisionmaking-for-cardiac-mri-5d-model-of-the-descending-aorta-for-the-detection-of-severestenosis

\section{Hosted file}

Figure6. docx available at https://authorea.com/users/351106/articles/475773-medical-decisionmaking-for-cardiac-mri-5d-model-of-the-descending-aorta-for-the-detection-of-severestenosis

\section{Hosted file}

Figure7 . docx available at https://authorea.com/users/351106/articles/475773-medical-decisionmaking-for-cardiac-mri-5d-model-of-the-descending-aorta-for-the-detection-of-severestenosis

\section{Hosted file}

Figure8. docx available at https://authorea.com/users/351106/articles/475773-medical-decisionmaking-for-cardiac-mri-5d-model-of-the-descending-aorta-for-the-detection-of-severestenosis 


\section{Hosted file}

Figure9. docx available at https://authorea.com/users/351106/articles/475773-medical-decisionmaking-for-cardiac-mri-5d-model-of-the-descending-aorta-for-the-detection-of-severestenosis

\section{Hosted file}

Figure10.docx available at https://authorea.com/users/351106/articles/475773-medicaldecision-making-for-cardiac-mri-5d-model-of-the-descending-aorta-for-the-detectionof-severe-stenosis 